\title{
Estudio del proceso de secado de fresa usando horno microondas \\ Study of the stramberry drying process using microwave dryer
}

\author{
María Bernarda Alvarado Bawab \\ M.Sc. Ingeniería - Ingeniería Química, Docente Tiempo Completo Ocasional, Institución Universitaria \\ ITSA, Barranquilla-Colombia. \\ E-mail: maryalvaradoiqad@gmail.com
}

\section{RESUMEN}

En esta investigación se evaluó el proceso y rendimiento del secado en horno microondas de 10kW de potencia a 2,45GHz empleando fresa para los experimentos. El horno consta de cuatro unidades compuestas por 4 magnetrones cada una que encienden y apagan de forma automatizada para mantener la potencia deseada en la cámara de secado, en cada uno de los experimentos se procesó $0,4 \mathrm{~kg}$ de muestra dispuesto en una bandeja de aluminio; durante el proceso se midió la pérdida de peso, la temperatura en tiempo real con cinco termómetros infrarrojos instalados dentro de la cámara de secado, el consumo energético y las condiciones ambientales de temperatura y humedad relativa. Las muestras tratadas poseían un contenido de humedad alrededor del $90 \%$ y se logró remover un $85 \%$ del peso de ésta durante el proceso, asumiendo que los demás compuestos de la fresa no varían. Se encontró que se pueden alcanzar rendimientos de $0,45 \mathrm{~kg} / \mathrm{kWh}$ durante los primeros 15 minutos de proceso alcanzando temperaturas alrededor de los $335 \mathrm{~K}$ y removiendo el $60 \%$ de la humedad contenida.

Palabras clave: Fresa; Secado; Microondas; Humedad; Energía; Rendimiento.

\begin{abstract}
This research evaluated the drying process and performance in a microwave oven with $10 \mathrm{~kW}$ of power at $2,45 \mathrm{GHz}$ using strawberry for experiments. The oven consists of four units composed of 4 magnetrons each that turn on and off in an automated way to maintain the desired power in the drying chamber; in each one of the experiments was processed $1 \mathrm{~kg}$ of sample placed on an aluminum tray. during the process the weight loss, the real-time temperature was measured with five infrared thermometers installed inside the drying chamber, the energy consumption and the environmental conditions of temperature and relative humidity. The treated samples had a moisture content of about $90 \%$ and it was possible to remove $85 \%$ of the weight during the process, assuming that the other strawberry compounds did not vary. It was found that yields of $0.45 \mathrm{~kg} / \mathrm{kWh}$ can be achieved during the first 15 minutes of processing reaching temperatures around $335 \mathrm{~K}$ and removing $60 \%$ of the contained moisture.
\end{abstract}

Keywords: Strawberry; Dry; Microwave; Moisture; Energy; Yield. 


\section{INTRODUCCIÓN}

Las aplicaciones de la energía de microondas han sido ampliamente estudiadas en las últimas décadas en un amplio espectro, donde los resultados reportan una exitosa mejora en los resultados de los experimentos con respecto a los métodos convencionales. La mayoría de los estudios llevados a cabo con energía de microondas se han centrado principalmente en su capacidad de calentamiento debido a la transferencia directa hacia el material que permite un calentamiento rápido y volumétrica de la muestra, mientras evita complicaciones como los amplios gradientes de temperatura entre la superficie y el interior tal como se presenta en los métodos convencionales[1].

El calentamiento volumétrico de las muestras va acompañado por la capacidad de la energía de microondas para penetrar el interior de la muestra e inducir un calentamiento más profundo en la muestra. Lo anterior se debe a que la energía de microondas a través de los campos eléctricos y magnéticos influye en las muestras, las moléculas polares e iónicas presentes tratan de alinearse con el campo eléctrico oscilante, lo que genera calor por fricción debido a que los gradientes iónicos cambian constantemente y las partículas colisionan [2].

La capacidad de los materiales para responder a los campos de electromagnéticos del orden de las microondas depende de su constante dieléctrica; lo que hace efectivo el calentamiento selectivo de muestras heterogéneas con componentes que poseen diferentes valores de esta propiedad física, provocando un incremento de la velocidad de calentamiento [3, 4].

Entre las aplicaciones industriales que se describen a continuación se encuentra el secado [5], la pasteurización, la cocción, procesos que generan cambios físicos en materiales, la sinterización de cerámicos, el curado, catálisis de reacciones, regeneración de materiales, etc.

La fresa es atractiva al consumidor por sus características sensoriales y nutricionales, pero su alto contenido de humedad la hace una fruta muy susceptible al deterioro por microorganismos [6], llegando a tener una vida útil a condiciones ambientales alrededor de 2 a 3 días después de la cosecha [7]. La industria agroalimentaria aplica tecnologías como la liofilización para obetner alimentos en polvo de gran calidad adicionando solutos como goma arábiga o maltodextrina [8]. Estudios de secado sin adicion de solutos se han realizado para la fresa en horno a $45^{\circ} \mathrm{C}$ con aire a $6 \mathrm{~m} / \mathrm{s}$ reportando que es posible secar muestras de $89 \%$ de humedad hasta que contengan un $8 \%$ en 5 horas de operación manteniendo un sabor agradable [9].
El secado por convección es un método ampliamente utilizado para aumentar la vida útil de un gran número de alimentos con contenidos en humedad superiores al $80 \%$, como es el caso de hortalizas y frutas. No obstante, dependiendo de las condiciones del proceso pueden producirse cambios físicos, químicos $\mathrm{y}$ físicoquímicos en los constituyentes de los productos deshidratados, alterándose consecuentemente su calidad. Con la finalidad de reducir la pérdida de compuestos nutritivos y bioactivos se están estudiando métodos emergentes de procesado, entre ellos el secado asistido con ultrasonido. En general, se ha visto que los ultrasonidos de potencia incrementan la velocidad de secado, siendo mayor el efecto cuando se aplican velocidades de aire inferiores a $5 \mathrm{~m} / \mathrm{s}$ y bajas temperaturas de proceso como lo describe Riera et al. (2011). En un estudio aplicando ultrasonido de potencia al secado convectivo de fresas se encontró que hay incrementos significativos en la capacidad de rehidratación en función de la potencia de radiación (0, 30 y $60 \mathrm{~W}$ ) en el secado a $313 \mathrm{~K}$, lo cual no se evidenció a 343K. Además, se logró una elevada retención de la vitamina $\mathrm{C}$ contenida [10].

La comparación de diferentes técnicas de secado como pretratamiento para la obtención de hojuelas de mango fue realizada evaluando los cambios originados en cuanto a la pérdida de color y otros atributos sensoriales, la pérdida de carotenoides y el tiempo de procesamiento. Dicho estudio arrojó que el pretratamiento de deshidratación osmótica (solución de sacarosa de $65^{\circ}$ Brix por 60 min y temperatura entre $37-40^{\circ} \mathrm{C}$ ) combinado con microondas (560W durante $7 \mathrm{~min}$ a una frecuencia de $2450 \mathrm{MHz}$ ) arroja una extracción porcentual de humedad en las hojuelas mucho mayor reduciendo los tiempos de secado al sol y en estufa. De igual forma, reducir los tiempos de exposición favorece el mantenimiento de las caracteristicas organolepticas de las hojuelas de mango [11].

Sin embargo, no se han reportado estudios de secado de fresa en horno microondas tipo túnel con banda transportadora continua de alta potencia $(10 \mathrm{~kW})$ como se presenta en este estudio donde se evalúa la tasa de secado y el rendimiento energético a lo largo del proceso.

La determinación de las condiciones de operación para el secado de frutas es de vital importancia ya que estas influyen en la estructura, color y propiedades fisicoquímicas en la deshidratación y rehidratación. En el estudio de Contreras (2006) se estudió la influencia de la temperatura del aire de secado de la aplicación de microondas y el pretratamiento de impregnación a vacío o deshidratación osmótica. En dicho estudio se encontró que la aplicación de microondas al secado por aire caliente provoca una disminución del tiempo 
de proceso y lleva asociado un cambio mayor de la solubilidad de las pectinas y mayores temperaturas con respecto al secado con aire seco a $50^{\circ} \mathrm{C}$ en muestras de manzana y fresa. Así mismo se induce a un aumento de la luminosidad de la fresa asociado a la disminución de antocianinas [12].

\subsection{Secado asistido por microondas}

La naturaleza de la energía de microondas para calentar selectivamente materiales dieléctricos hace evidente su uso en el secado de materiales. La mayoría de las investigaciones en esta aplicación se centran en la preservación de los alimentos como las pastas, frutas, verduras y aperitivos salados a través de la deshidratación [13-15].

En estos procesos se ha encontrado que el secado asistido por microondas ofrece ventajas sobre los secadores convencionales de aire caliente, tales como: los tiempos de secado más cortos, el aumento de la eficiencia energética y el potencial de la reducción del tamaño de los equipos de secado requeridos [13]. De igual forma, se hace interesante y aún más eficaz la combinación con procesos convencionales como el secado con aire caliente [14]. Sin embargo, es necesario realizar pruebas experimentales para definir los rangos de operación y el suministro adecuado de la radiación con microondas.

La deshidratación de frutas y verduras es un proceso muy conveniente para optimizar la vida útil, manteniendo el valor nutritivo y la calidad. Las características comúnmente elegidas para rechazar el secado asistido por microondas de frutas y verduras son generalmente el tiempo de secado y la calidad del producto seco (textura, dureza, color de la superficie, la concentración de vitaminas, la capacidad de rehidratación, y lo más importante, el sabor). Un sistema de secado por microondas automático y continuo fue empleado por Li, et al. (2010), para controlar la temperatura de la muestra y medir la masa de muestras durante el secado de manzana. El estudio se llevó a cabo empleando tres temperaturas de secado $(348,338,328 \mathrm{~K})$. Se encontró que este método de automatización asegura un mejor control de temperatura y un menor consumo de energía [14].

Una investigación sobre el uso potencial de secado asistido por microondas de aperitivos salados estudia la eficacia del proceso y la calidad del producto en comparación con métodos convencionales. Muchos investigadores encontraron que el uso de muestras predeshidratadas mejora la eficiencia global del proceso, lo que indica que el contenido inicial de humedad es un factor que influye en los procesos de secado asistido por microondas y puede afectar la calidad del producto seco [15]. Otro resultado interesante fue la calidad del producto, que consiste en ser aún más seco y uniforme a través de toda la muestra; lo anterior se logró cuando hubo períodos de descanso durante el tiempo de procesamiento de microondas.

Zhang et al. (2007), obtuvieron una calidad uniforme del producto cuando se utiliza un intervalo de un minuto durante el tiempo del calentamiento por microondas; es decir, 12 segundos de irradiación, un minuto de descanso en el horno, y posteriormente un nuevo encendido de irradiación durante 12 segundos. Se sugirió que estas condiciones de funcionamiento eliminan los gradientes de temperatura a lo largo de la muestra [16].

Holtz, et al. (2010), estudiaron el comportamiento del secado, el cambiodela temperatura, la higroscopicidad, propiedades dieléctricas y el consumo específico de energía de materiales sólidos y porosos (colza, patata, pan y hormigón ligero) durante la exposición en un horno microondas por convección, debido a que estos presentan diferentes propiedades y presentan diferentes tasas de secado. Los resultados indican que una relación entre la profundidad de penetración de microondas y la actividad del agua de los materiales influían en el proceso. El secado de los materiales alimenticios estaba limitado por la combustión ya sea debido a la temperatura en la región higroscópica o debido al desarrollo de puntos calientes, posiblemente inducidos por la estructura del material [17].

\section{METODOLOGÍA}

Para el desarrollo de este trabajo se utilizó un horno capaz de suministrar una radiación microondas a una frecuencia de $2,45 \mathrm{GHz}$ con potencia de $10 \mathrm{~kW}$ distribuidos en 12 magnetrones ubicados a lo largo y ancho de la cámara de secado. Dicha frecuencia es la característica para los procesos industriales que requieren adición de calor a materiales. Para el secado de la fresa, inicialmente se cortó la muestra en trozos de $1 \mathrm{~cm} 3$ aproximadamente, luego se dispuso en bandejas de aluminio rectangulares $(200 \mathrm{~mm} \times 120$ $\mathrm{mm}$ ) asegurándose de no ubicar un trozo sobre otro. La masa de la fruta en cada bandeja fue de $400 \mathrm{~g}$. Posteriormente, las bandejas se ubicaron en la cámara de secado registrando su peso cada 5 min durante 45 min. La prueba se dio por terminada una vez se llegó a una remoción aproximada del $80 \%$, valor considerado como la humedad total contenida en la fresa. En cada intervalo se registró el consumo de energía, la temperatura alcanzada por la muestra utilizando un termómetro infrarrojo, la humedad relativa y la temperatura ambiente.

La cámara de secado contiene supresores a cada lado con el objeto de prevenir fugas a densidades superiores de $5 \mathrm{~mW} / \mathrm{cm} 2$. Un extractor de aire para 
mantener refrigerados los equipos generadores de la radiación microondas, y un extractor de vapor en la cámara de secado que remueve el vapor generado durante el proceso en la cámara.

La perdida de humedad de la fresa $(\% \mathrm{H})$ se calculó empleando la ecuación 1 con los datos obtenidos en la medición del peso de las muestras al inicio de la prueba, en diferentes intervalos de tiempo y al final del proceso:

$$
\% H=\frac{\left(W_{i}-W_{f}\right)}{W_{i}} \times 100 \%
$$

Donde, $\% \mathrm{H}$ es el porcentaje de humedad, $\mathrm{w}_{\mathrm{i}}$ es la masa de la muestra al inicio del proceso en $\mathrm{kg}_{ } \mathrm{w}_{\mathrm{f}}$ es la masa de la muestra en un tiempo determinado en $\mathrm{kg}$.

Para el cálculo del rendimiento del secado se calculó la diferencia en la perdida de peso en un intervalo de tiempo constante de 5 minutos y se dividió en el consumo energético registrado por el contador instalado para tal fin como se presenta en la ecuación 2.

$$
\eta=\frac{W_{i}-W_{f}}{E}
$$

Donde, $\eta$ es el rendimiento del secado en $\mathrm{kg} / \mathrm{kWh}$ y E es la energía consumida en kWh.

\section{RESULTADOS Y DISCUSIÓN}

Durante la prueba la humedad relativa estuvo entre el 48 y $64 \%$ y la temperatura ambiente entre 296 y
300K. Los datos obtenidos luego de la realización de las corridas experimentales se presentan en la tabla 1 .

Con la información obtenida fue posible evaluar la homogeneidad del proceso a través de la cámara de secado, calcular el rendimiento energético del proceso, y construir las curvas de secado. En la figura 1 se presenta la pérdida de humedad de la fresa a través del tiempo para cada una de las bandejas introducidas en el horno microondas.

En la figura 1 se puede observar el porcentaje de humedad de las fresas a través del tiempo; en ella se nota como al inicio de la remoción de humedad se da de forma inmediata lo cual se debe a que el calentamiento con microondas se da en forma volumétrica e inmediatamente las moléculas polares contenidas en el material entran en contacto con la radiación microondas. Una velocidad de secado constante y alta se presentó hasta el minuto 20 donde se logró remover el $70 \%$ de la humedad contenida en la fresa y posteriormente se presenta una zona de remoción lenta debido a que la ausencia del agua que limita la absorción de la energía transportada por la radiación microondas y por consiguiente, se da un secado más lento. Por otra parte, se observa que la remoción de humedad es homogénea a lo largo de la cámara de secado y en todas se alcanza el mismo contenido de humedad final para el producto.

Así como lo reportó Wang en el 2013 en el estudio de la uniformidad del secado de lechuga por pulsos en horno microondas con vacío donde se lograba una reducción del tiempo de procesamiento en un 50\% comparado con el secador microondas convencional. Además, se

Tabla 1. Resultados experimentales.

Table 1. Experimental Results.

\begin{tabular}{|c|c|c|c|c|}
\hline $\begin{array}{c}\text { Tiempo } \\
(\mathbf{m i n})\end{array}$ & $\begin{array}{c}\text { Porcentaje de } \\
\text { humedad perdida } \\
\mathbf{( \% )}\end{array}$ & Temperatura (K) & $\begin{array}{c}\mathbf{k W h} \\
\text { consumido }\end{array}$ & Rendimiento (kg/kWh) \\
\hline 0 & 0,00 & 287 & 0,0 & 0,000 \\
\hline 5 & 14,46 & 333 & 1,1 & 0,197 \\
\hline 10 & 35,04 & 337 & 1,1 & 0,450 \\
\hline 15 & 55,80 & 331 & 1,1 & 0,453 \\
\hline 20 & 71,38 & 322 & 1,1 & 0,340 \\
\hline 25 & 77,15 & 323 & 1,1 & 0,126 \\
\hline 30 & 81,20 & 320 & 1,1 & 0,088 \\
\hline 35 & 84,63 & 313 & 1,1 & 0,068 \\
\hline 40 & 86,33 & 317 & 1,0 & 0,047 \\
\hline 45 & 87,71 & 323 & 1,1 & 0,032 \\
\hline
\end{tabular}


logra reducir la decoloración de las hojas de lechuga y se presenta alta capacidad de rehidratación y dureza después de la rehidratación [18].

Figura 1. Curva de secado de fresa en cubos de $1 \mathrm{~cm}^{3}$ en horno túnel microondas a 10kW.

Figure 1. Cubed strawberry drying curve $1 \mathrm{~cm}^{3}$ in tunnel microwave oven at $10 \mathrm{~kW}$ of power.

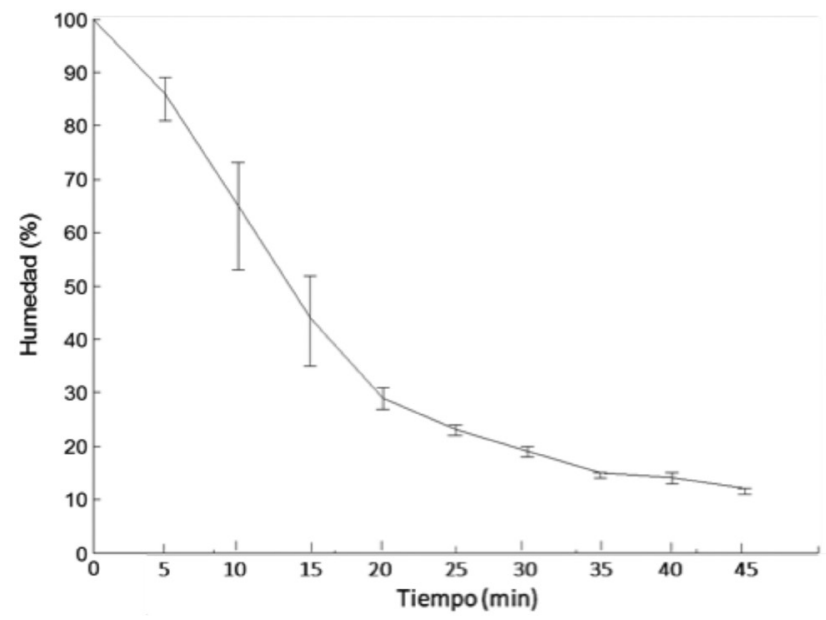

En la figura 2 se presenta la temperatura alcanzada en cada una de las bandejas durante el proceso de secado con microondas.

Figura 2. Temperatura alcanzada en el proceso de secado de fresa en horno microondas túnel a $10 \mathrm{~kW}$.

Figure 2. Temperature reached by strawberry at microwave tunnel oven drying process at $10 \mathrm{~kW}$ of power.

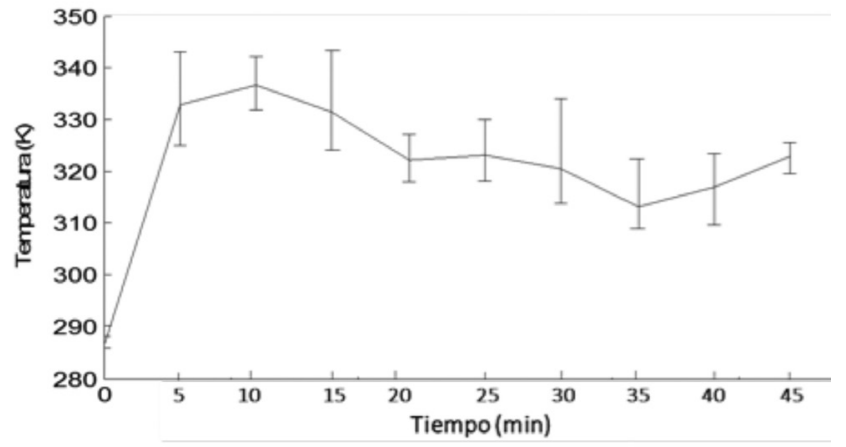

En la figura 2 se puede observar que la temperatura de las fresas se incrementa rápidamente hasta alrededor de $333 \mathrm{~K}$ donde se mantiene hasta el minuto 15 donde empieza a decaer hasta alrededor de $323 \mathrm{~K}$ donde se mantiene hasta finalizar la prueba. Lo anterior se presenta debido a que el contenido de humedad en el producto es quien determina la capacidad de absorción de la energía transportada por la radiación microondas, los fenómenos convectivos, difusivos y la porosidad dominan la disipación del calor trayendo como consecuencia la curva presentada anteriormente. El rendimiento fue calculado en términos de los kilogramos de agua total evaporada en cada intervalo de tiempo con respecto al consumo de energía eléctrica y se muestra en la figura 3.

Figura 3. Rendimiento del proceso de secado de fresa en microondas túnel a $10 \mathrm{~kW}$ de potencia.

Figure 3. Microwave drying process yield in oven continuous tunnel-type of strawberry with $10 \mathrm{~kW}$ of power.

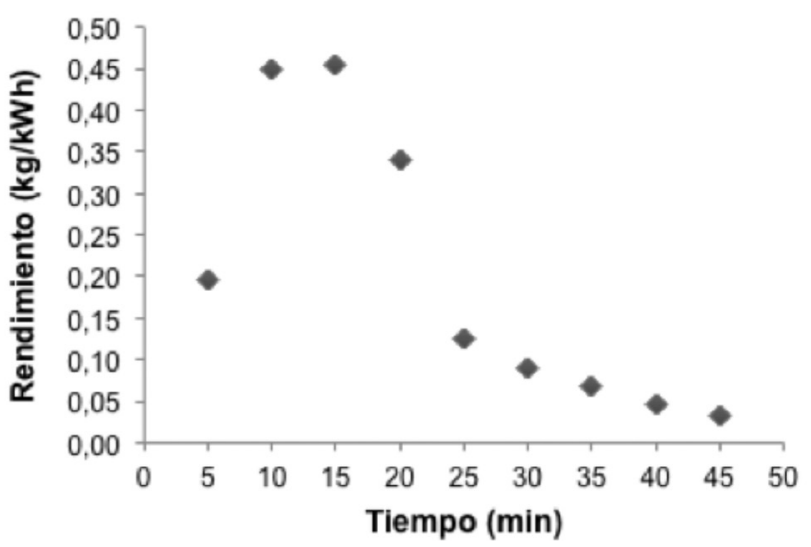

La figura 3 presenta que el mayor aprovechamiento de la energía se obtiene entre el minuto 5 y 20 donde el rendimiento es entre 0,3 y $0,45 \mathrm{~kg} / \mathrm{kWh}$. Lo anterior sustenta que hasta 20 minutos es la zona favorable para realizar un secado efectivo con microondas.

\section{CONCLUSIONES}

El agua es el componente mayoritario en la fresa y aplicando una radiación microondas, con una potencia de $10 \mathrm{~kW}$ a $2,45 \mathrm{GHz}$ durante 45 minutos, sobre trozos de estas es posible reducir en un $85 \%$ el peso de la muestra removiendo el agua contenida. La temperatura alcanza un valor alrededor de $335 \mathrm{~K}$ durante los primeros 15 minutos del proceso donde se remueve la mayor cantidad de agua $(60 \%)$, posterior a esto la temperatura disminuye manteniéndose alrededor de los 320K. Lo anterior, se explica ya que, al remover el agua, la cantidad de compuestos absorbedores de la energía contenida en las ondas electromagnéticas se reducen presentando además la disminución del rendimiento energético para lograr el secado. En el proceso de secado se alcanzó rendimientos de 0,45 kilogramos de agua removida por cada $\mathrm{kWh}$ consumida el cual se reduce luego de los 15 minutos de operación en función de la perdida de humedad de la muestra. 
Mediante la observación de los productos finales se notó diferencias sensoriales en los productos, tales como el brillo, dulzor, textura, entre otros, que afectan su calidad, estructura, color y son de gran interes para estudios posteriores.

\section{REFERENCIAS}

[1] J. M. Bélanger, J. R. Jocelyn Paré, O. Poon, C. Fairbridgem S. Ng, S. Mutyala, R. Hawkins, "Remarks on Various Applications of Microwave Energy". Journal of the Microwave Power and Electromagnetic Energy, 42 (4), 24-44, 2008.

[2] M. B. Alvarado, (2012). Estudio de secado en horno continuo microonda del carbón de Bijao-Córdoba. Tesis de Maestría, Universidad Nacional de Colombia.

[3] S. Marland, A. Merchant, B. Han and N. Rowson, "Dielectric Properties of coal," Fuel, 79, 1283-1288, 2001.

[4] J. Monzó-Cabrera, A. Díaz-Morcillo, J. M. CataláCivera, and E. de los Reyes, "Effect of Dielectric Properties on Moisture Leveling in MicrowaveAssisted Drying of Laminar Materials," Microwave and Optical Technology Letters, 30, 165-168, 2001.

[5] M.B. Alvarado, J. Mejía, M. Vanegas-Chamorro, L. Hernández, "Influential variables in coal batch microwave drying". Alternative Energies and Energy Quality (SIFAE), 2012 IEEE International Symposium on. $1-6.2012$.

[6] G. Moraga, N. Martínez-Navarrete, A. Chiralt . "Water sorption isotherms and glass transition in strawberries: influence of pretreatment", Journal of Food Engineering, 62(4), 315-321, 2004.

[7] FAO-PRODAR. Fichas Técnicas de Procesados de Frutas. 2014 [En linea], [consulta, 13 de marzo del 2016]. Disponible desde: http://www.fao.org/3/aau168s.pdf

[8] M. Tarín, E. García, (2015). Evaluación de la calidad funcional de extractos de mora y fresa liofilizada. Tesis fin de carrera Ciencia y Tecnología de los Alimentos, Universidad Politécnica de Valéncia.

[9] L. Ruiz, L. Castro, M. Quicazán. “Influencia de las condiciones de secado en el color de fresas variedad Chandler". Encuentro Nacional de Investigación y Desarrollo (ENID), 2015 Universidad Nacional de Colombia. Manizales. 1-4. 2015.
[10] Gamboa-Santos, J., Montilla, A., Villamiel, M. García-Pérez, J. Aplicación de ultrasonidos de potencia al secado convectivo de fresas. VII Congreso Español de Ingeniería de los Alimentos (cesia), 2012. Universidad de Castilla-La Mancha. Madrid. 1-11. 2012.

[11] M. García-Paternina, A. Alvis-Bermudez, C. García-Mogollón, "Evaluación de los Pretratamientos de Deshidratación Osmótica y Microondas en la Obtención de Hojuelas de Mango (Tommy Atkins)", Información tecnológica. 26(5) 63-70, 2015.

[12] C. Contreras Monzón, (2006). “Influencia del método de secado en parámetros de calidad relacionados con la estructura y el color de manzana y fresa deshidratadas". Tesis Doctoral. Universidad Politécnica De Valencia.

[13] A. Altan and M. Maskan, "Microwave Assisted Drying for Short-Cut (Ditalini) Macaroni: Drying Characteristics and Effect of Drying Processes on Starch Properties". Food Research International., 38, 787-796, 2005.

[14] L. Zhenfeng, G.S.V. Raghavanb, V. Orsatb, "Optimal power control strategies in microwave drying", Journal of Food Engineering., 99(3), 263-268, 2010.

[15] J. Zhang, M. Zhang, L. Shan, and Z. Fang, "Microwave-Vacuum Heating Parameters for Processing Savory Crisp Bighead Carp (Hypophthalmichthys nobilis) Slices"., Journal of Food Engineering, 79(3), 885-891, 2007.

[16] M. N. Berteli, and A. Marsaioli, "Evaluation of Short Cut Pasta Air Dehydration Assisted by Microwaves as Compared to the Conventional Drying Process"., Journal of Food Engineering, 68(2), 175-183, 2005.

[17] E. Holtz, L. Ahrné, M. Rittenauer, A. Rasmuson, "Influence of dielectric and sorption properties on drying behaviour and energy efficiency during microwave convective drying of selected food and non-food inorganic materials"., Journal of Food Engineering, 97(2), 144-153, 2010.

[18] Y. Wang, M. Zhang, A. Mujumdar, K. Mothibe, S. Roknul Azam, "Study of Drying Uniformity in Pulsed Spouted Microwave-Vacuum Drying of Stem Lettuce Slices with Regard to Product Quality"., Drying Technology, 31(1), 91-101, 2013. 